\title{
A Multi-wavelength MOCASSIN model of the Magellanic-type galaxy NGC 4449
}

\author{
O. Ł. Karczewski ${ }^{1}$, M. J. Barlow ${ }^{1}$, M. J. Page ${ }^{2}$ and S. C. Madden ${ }^{3}$ \\ ${ }^{1}$ Department of Physics and Astronomy, University College London, \\ Gower Street, London, WC1E 6BT, UK \\ ${ }^{2}$ Mullard Space Science Laboratory, University College London, \\ Holmbury St. Mary, Dorking, RH5 6NT, UK \\ ${ }^{3}$ CEA Saclay Service d'Astrophysique, L'Orme des Merisiers Bat 709, \\ 91191 Gif-sur-Yvette Cedex, France \\ e-mail: olk@star.ucl.ac.uk
}

\begin{abstract}
We use the photoionisation and dust radiative transfer code MOCASSIN to create a model of the dwarf irregular galaxy NGC 4449. The best-matching model reproduces the global optical emission line fluxes and the observed spectral energy distribution (SED) spanning wavelengths from the UV to sub-mm, and requires the bolometric luminosity of $6.25 \times 10^{9} L_{\odot}$ for the underlying stellar component, $M_{\mathrm{dust}} / M_{\mathrm{gas}}$ of $1 / 680$ and $M_{\mathrm{dust}}$ of $2.2 \times 10^{6} M_{\odot}$.
\end{abstract}

Keywords. dust, extinction, galaxies: dwarf, galaxies: general, galaxies: individual (NGC 4449), galaxies: stellar content, methods: numerical

\section{Introduction}

The spectral energy distribution (SED) of a galaxy emerges as a combination of the underlying stellar emission, the degree of reprocessing taking place in the interstellar medium (ISM) and thermal or non-thermal emission due to dynamical or evolutionary processes. As a consequence, galaxies emit across the entire range of the electromagnetic spectrum and the distribution of the energy output as a function of wavelength provide clues to the galaxy's past and present. Numerical models that reproduce the observed global properties of a galaxy over a wide range of wavelengths can be useful in understanding the physical and chemical nature of the underlying stellar and dust components. Here we present a MOCASSIN model of the dwarf irregular galaxy NGC 4449.

MOCASSIN (Ercolano et al. 2003, 2005, 2008) is a fully 3-dimensional photoionisation and dust radiative transfer code. Recent applications of the code include modelling the planetary nebula NGC 6302 (Wright et al. 2011) and studying dust formation by SN 2008S (Wesson et al. 2010). Modelling entire galaxies is technically challenging due to their large physical sizes and the high level of complexity of the galactic environments. Dwarf galaxies are ideal candidates due to their intrinsically small sizes, relatively simple star formation histories and the low degree of processing of their interstellar material.

NGC 4449 (Fig. 1) is a metal-poor magellanic-type irregular galaxy. It is not a typical dwarf irregular: at the distance of $4.21 \mathrm{Mpc}$ its $\mathrm{H}$ I envelope extends to a radius of $14.2 \mathrm{kpc}\left(11^{\prime} .6\right)$, equal to approximately $6 R_{25}$ (Swaters et al. 2002), and exhibits a counter-rotating core. It was selected for this study for its extensive coverage of photometric measurements for wavelengths ranging from the UV to sub-mm. Recently, new FIR observations were acquired by the Herschel Space Observatory (Pilbratt et al. 2010) as part of the Guaranteed Time Key Project: Dwarf Galaxy Survey (PI: Suzanne Madden). 


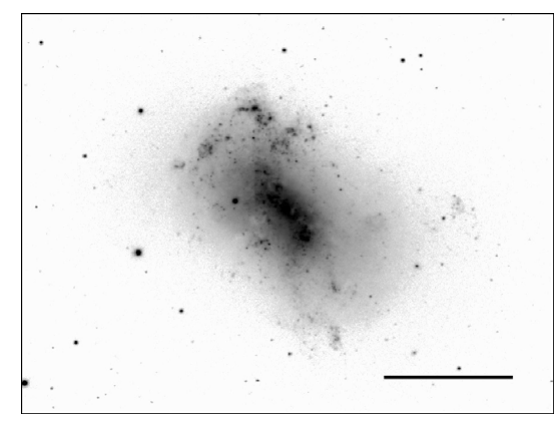

Figure 1. SDSS $r$-band image of NGC 4449. North is up, east is to the left. The image is centred at $12^{\mathrm{h}} 28^{\mathrm{m}} 11^{\mathrm{s}} .1,+44^{\circ} 05^{\prime} 37^{\prime \prime}(\mathrm{J} 2000)$. The bar is $2^{\prime}$ in length.

The emission line fluxes, used to constrain the models, were derived from global spectra, acquired with the drift scanning technique, and are representative of the entire galaxy (Kobulnicky et al. 1999).

\section{Methods and Results}

Our MOCASSIN simulations use 0.5 million grid cells to establish the observed H I profile of Swaters et al. (2002) within the inner $3.3 \mathrm{kpc}(2.7)$, enclosing the visible part of NGC 4449 and about a half of its total H I mass. The geometry, gas density distribution and the observed abundances are fixed inputs. Trial families of simulations are run and a range of $M_{\text {dust }} / M_{\text {gas }}$, bolometric stellar luminosities $L_{\star}$ and input stellar radiation fields are tested. A parameter set providing the best match to the shape of the low-resolution SED, the global emission line fluxes and the integrated $\mathrm{H} \alpha$ luminosity (Hunter et al. 1999) is expanded into a new, higher resolution parameter space and the procedure is iterated until a satisfactory match with all observables is obtained. Fig. 2 gives a schematic summary of this method.

The stellar radiation field is the most crucial input, as it results from the assumed star formation history. In this work, two continuous episodes of constant star formation activity were assumed and an initial (and subsequently expanded, according to Fig. 2) set of 45 templates generated with STARBURST99 (Leitherer et al. 1999; version 201101-17) was tested. A small subset of the resulting SEDs is shown in Fig. 3.

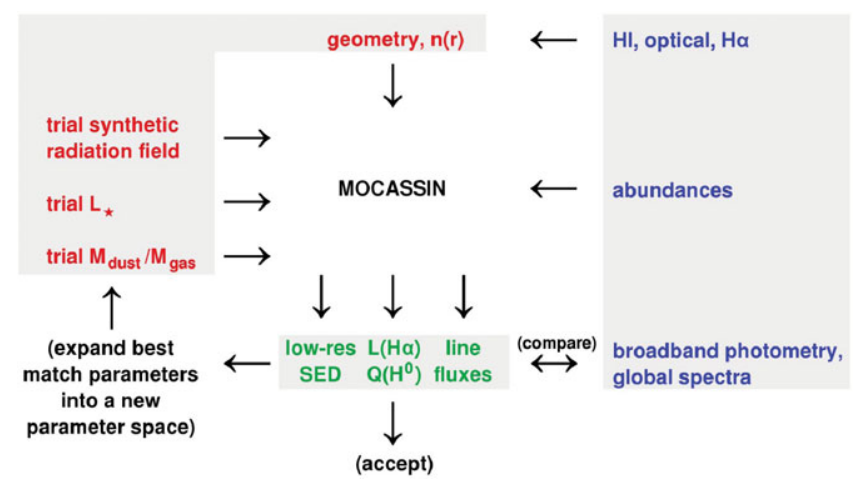

Figure 2. A schematic summary of the modelling method used. The shaded regions group together the inputs to MOCASSIN (left) the outputs (middle) and the observational constrains (right). 
To break the degeneracy it is helpful to ask if both (i) the shape of the observed SED and (ii) the total emergent $L(\mathrm{H} \alpha)$ for a particular star formation scenario can be matched by varying $M_{\text {dust }} / M_{\text {gas }}$ and/or $L_{\star}$, whose effect on the resulting SED is relatively simple to predict. This condition allows for more than 90 per cent of the initial templates to be rejected at an early stage. The final best-matching model is selected by inspecting the predicted emission line fluxes. For NGC 4449 the best-matching SED is presented in Fig. 4 and the derived physical quantities are given in Table 1.

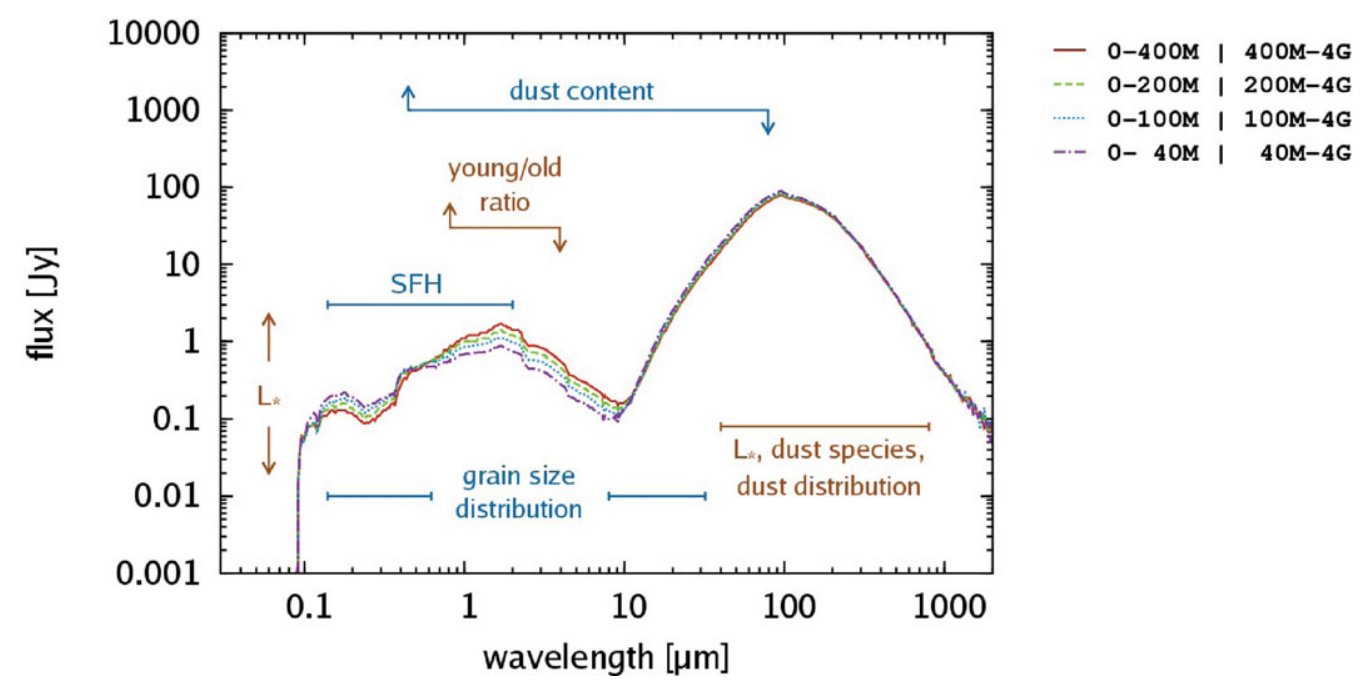

Figure 3. A family of predicted SEDs for four trial star formation scenarios assuming two episodes of continuous star formation with two different star formation rates. All input parameters are identical except for the length of the two star formation episodes (see legend for details). The approximate wavelength ranges affected by the various free parameters are indicated.

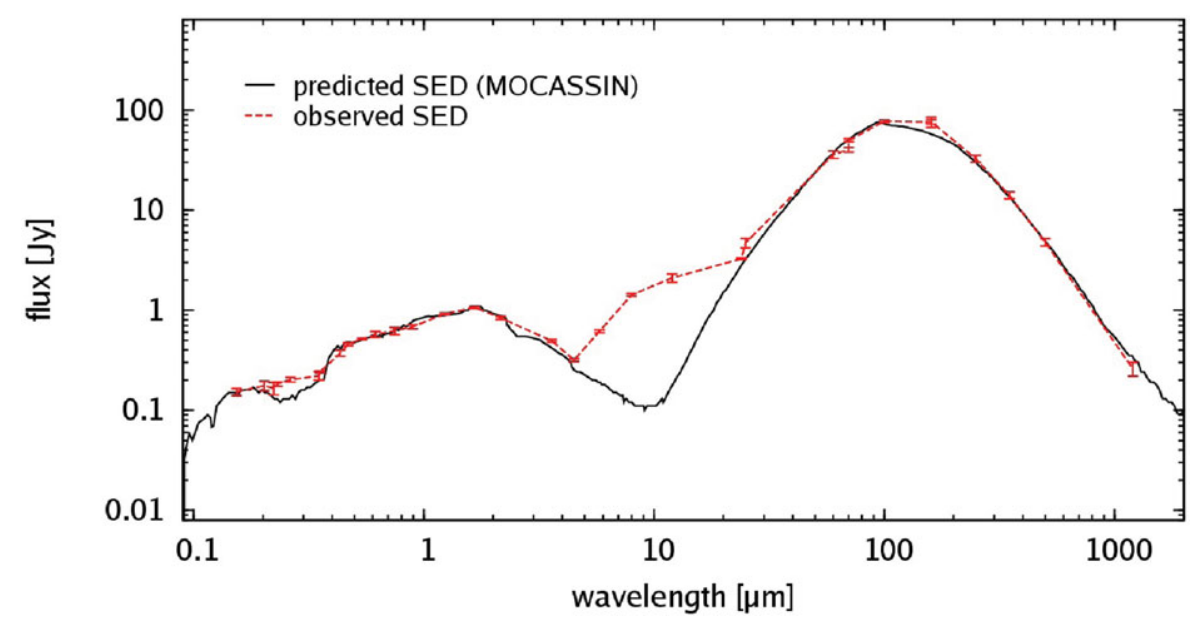

Figure 4. The predicted SED of the best-matching MOCASSIN model of NGC 4449. PAHs are not included in this model. The observed SED is based on the archive data (GALEX in the UV, Swift in the UV/optical, SDSS in the optical, 2MASS in the NIR) and the published photometry (Engelbracht et al. 2008, Hunter et al. 1986, Böttner et al. 2003; at longer wavelengths). In the FIR new Herschel PACS and SPIRE measurements are included (Remy et al., in prep.). 


\begin{tabular}{lc}
\hline parameter & MOCASSIN model of NGC 4449 \\
\hline$L_{\star}$ & $6.25 \pm 0.25 \times 10^{9} L_{\odot}$ \\
$M_{\star}$ & $1.2 \pm 0.1 \times 10^{9} M_{\odot}$ \\
$M_{\text {gas }}$ & $1.5 \pm 0.2 \times 10^{9} M_{\odot}$ \\
$M_{\text {dust }}$ & $2.2 \pm 0.2 \times 10^{6} M_{\odot}$ \\
$M_{\text {dust }} / M_{\text {gas }}$ & $1 / 680(1 / 850$ to $1 / 540)$ \\
dust composition & $100 \%$ amorphous carbon \\
dust grain sizes & $0.005-1 \mu \mathrm{m}, n \propto a^{-3.5}$ \\
SF episodes & $6 \pm 2$ Gyr to $120 \pm 40 \mathrm{Myr} 120 \pm 40 \mathrm{Myr}$ to present \\
relative $M_{\star}$ & $120 \quad 0.06$ to $0.12 M_{\odot} \mathrm{yr}^{-1}$ \\
$<$ SFR $>$ & 0.2 to $0.3 M_{\odot} \mathrm{yr}^{-1}$ \\
\hline
\end{tabular}

Table 1. Summary of global parameters derived from the best-matching MOCASSIN model of NGC 4449 assuming two continuous episodes of star formation. The global emission line fluxes are matched to within 20 per cent or better, with the exception of the sulphur lines [S II] $\lambda \lambda 6717,31$, which are overpredicted by a factor of four.

\section{Discussion}

The gas and dust masses derived from our MOCASSIN model of NGC 4449 are consistent with earlier estimates (Hunter et al. 1999; Engelbracht et al. 2008). Our bestmatching star formation scenario broadly agrees with the presence of an old (3-5 Gyr) and a young population of stars, and continuous star formation over the last $1 \mathrm{Gyr}$ (Bothun 1986; Martin \& Kennicutt 1997; Annibali et al. 2008). However, the predicted present-day star formation rate is only one-fourth of the estimate based on the H $\alpha$ luminosity (Hunter et al. 1999). The discrepancy between these two independent estimates highlights the differences in the adopted initial mass function (IMF) as well as the limitations of the two-episode model.

The presented technique assumes spherical symmetry for NGC 4449 and a two-episode star formation history, both of which are great simplifications. Also, at present PAHs are not included in the models and, as a consequence, the $4-20 \mu \mathrm{m}$ range is not correctly predicted. Nevertheless, most of the global parameters are predicted to within the observational uncertainties, and therefore this technique may prove useful in studying the characteristics of dust and the underlying stellar components in other dwarf galaxies.

\section{References}

Annibali, F., Aloisi, A., Mack, J., et al. 2008, AJ, 135, 1900

Bothun, G. D. 1986, AJ, 91, 507

Ercolano, B., Barlow, M. J., Storey, P. J., \& Liu, X.-W. 2003, MNRAS, 340, 1136

Ercolano, B., Barlow, M. J., \& Storey, P. J. 2005, MNRAS, 362, 1038

Ercolano, B., Young, P. R., Drake, J. J., \& Raymond, J. C. 2008, ApJS, 175, 534

Böttner, C., Klein, U., \& Heithausen, A. 2003, A\&A, 408, 493

Engelbracht, C. W., Rieke, G. H., Gordon, K. D., et al. 2008, ApJ, 678, 804

Hunter, D. A., Gillett, F. C., Gallagher, III, J. S., Rice, W. L., \& Low, F. J. 1986, ApJ, 303, 171

Hunter, D. A., van Woerden, H., \& Gallagher, J. S. 1999, AJ, 118, 2184

Kobulnicky, H. A., Kennicutt, Jr., R. C., \& Pizagno, J. L. 1999, ApJ, 514, 544

Leitherer, C., Schaerer, D., Goldader, J. D., et al. 1999, ApJS, 123, 3

Martin, C. L. \& Kennicutt, Jr., R. C. 1997, ApJ, 483, 698

Pilbratt, G. L., Riedinger, J. R., Passvogel, T., et al. 2010, A\&A (Letters), 518, L1

Swaters, R. A., van Albada, T. S., van der Hulst, J. M., \& Sancisi, R. 2002, A\&A, 390, 829

Wesson, R., Barlow, M. J., Ercolano, B., et al. 2010, MNRAS, 403, 474

Wright, N. J., Barlow, M. J., Ercolano, B., \& Rauch, T. 2011, MNRAS, 418, 370 